Arq. Bras. Med. Vet. Zootec., v.64, n.1, p.63-66, 2012

\title{
Mielografia em ouriço-cacheiro (Sphiggurus villosus)
}

[Myelography in orange-spined-hairy-dwarf-porcupine (Sphiggurus villosus)]

\author{
N.T. Sant Anna ${ }^{1}$, B.S. Silva $a^{2}$, G.C. Soresini ${ }^{3}$, L.C.S. Silva ${ }^{4}$ \\ ${ }^{1}$ Viva Bicho Clínica Veterinária e Consultoria - Piracicaba, SP \\ ${ }^{2}$ Aluna de pós-graduação - Pontifícia Universidade Católica do Paraná - PUCPR, PR \\ ${ }^{3}$ Centro de Triagem de Animais Silvestres - Cetas - PUCPR/IBAMA, PR \\ ${ }^{4}$ Aluna de pós-graduação - Faculdade de Medicina Veterinária e Zootecnia - FMVZ-USP, SP
}

\section{RESUMO}

Relata-se o procedimento de mielografia em um ouriço-cacheiro Sphiggurus villosus, adulto, procedente de vida livre. Após o exame e com o animal sob anestesia geral, evidenciou-se um desvio dorsal do contraste na altura da sétima vértebra torácica $\left(\mathrm{T}_{7}\right)$, causado por compressão em região ventral dessa vértebra, sugestivo de edema ocasionado pela hemorragia intramedular secundária ao trauma sofrido pelo animal em vida livre. Assim sendo, a mielografia em ouriço-cacheiro mostrou ser bastante válida e eficiente ao apresentar como vantagens a maior precisão para delimitar e localizar a lesão presente na coluna vertebral.

Palavras-chave: roedor, radiografia contrastada, coluna vertebral

\begin{abstract}
The myelography procedure is reported in an adult of the Sphiggurus villosus (hairy-tree-porcupine) species coming from the wild, in the region of Curitiba/PR. After proceeding with the examination, while the animal was under general anesthesia, there was a dorsal deviation of the contrast in the height of the seventh thoracic vertebra $\left(T_{7}\right)$ caused by compression in the ventral region of the vertebra. This is suggestive of edema caused by intramedullary hemorrhage secondary to trauma suffered by the animal in the wild. Therefore, the use of myelography in Sphiggurus villosus (hairy-tree-porcupine) proved to be quite valid and efficient, presenting the advantages of greater precision to delimit and locate the lesion present in the vertebral column.
\end{abstract}

Keywords: rodents, contrast radiography, vertebral column

\section{INTRODUÇÃO}

O ouriço-cacheiro (Sphiggurus sp.) pertence à maior ordem da classe dos mamíferos, os roedores. Nesta classe, atualmente se encontram 2.021 espécies, 443 gêneros e 29 famílias (Wilson e Reeder, 1993), sendo o gênero Sphiggurus (F. Cuvier, 1823) composto por cinco espécies encontradas no Brasil. Na região Sul, são encontradas duas espécies, $S$. spinosus e S. villosus (Oliveira e Bonvicino, 2006).

O conhecimento da anatomia é fundamental para a avaliação das radiografias, sendo o desconhecimento um dos elementos que dificultam a interpretação dos exames de animais selvagens. Exames contrastados, como urografia excretora, uretrocistografia, fistulografia, mielografia, sinografia, peritoneografia, angiografia, traqueografia, broncografia e artrografia, também podem ser realizados em animais selvagens, todavia suas descrições na literatura ainda são escassas (Pinto, 2007).

A radiografia convencional é o método mais utilizado para obtenção de imagens neurológicas veterinárias, devido ao seu baixo custo e ampla disponibilidade, já a mielografia é o

Recebido em 9 de maio de 2011

Aceito em 21 de junho de 2011

E-mail: nathresant@hotmail.com 
procedimento de contraste que mais traz benefícios diagnósticos para a neuroradiologia (Slatter, 1998).

A mielografia é realizada após a efetuação do estudo radiográfico rotineiro da coluna vertebral e consiste na aplicação de meio de contraste à base de iodo no canal medular, iopamidol e iohexol, por exemplo. A introdução desse meio de contraste é feita no espaço subaracnoideo com o propósito de demonstrar lesão em medula espinhal, podendo ser intramedular, devido a tumores medulares ou edema de medula em decorrência de traumatismo, ou extramedular, devido à herniação de disco intervertebral, fratura ou luxação vertebral, ou tumores de origem extradural (Slatter, 1998; Kealy e McAllister, 2005; Knipe, 2007). As regiões de escolha para aplicação do meio de contraste são a lombar, entre a última vértebra lombar e a primeira sacral, ou a região da cisterna magna, entre o crânio e a primeira vértebra cervical, após assepsia prévia do local (Kealy e McAllister, 2005).

O exame pode confirmar a lesão vista ou suspeita em radiografias simples, definir a extensão da lesão, ou identificar pacientes que possam ser beneficiados por meio de procedimento cirúrgico (Thrall, 2002). A mielografia está contraindicada quando o diagnóstico é obtido mediante radiografia simples, ou quando as informações por ela obtidas não alterarem o curso do tratamento (Slatter, 1998), e em quadros de doença inflamatória (Kealy e McAllister, 2005).

Possíveis complicações deste exame podem decorrer em razão da neurotoxicidade, ainda que baixa, do contraste utilizado, causando convulsão após a realização do exame, apneia, arritmias cardíacas e sinais neurológicos associados ao traumatismo causado pela inadequada aplicação da agulha. A convulsão é facilmente revertida com o uso de diazepam intravenoso (Barone et al., 2002; Kealy e McAllister, 2005).

Este trabalho tem como objetivo relatar o procedimento de mielografia em um indivíduo adulto de ouriço-cacheiro procedente de vida livre.

\section{CASUÍSTICA}

Um exemplar adulto de ouriço-cacheiro (Sphiggurus villosus) com massa corpórea de
$1,3 \mathrm{~kg}$, proveniente da cidade de Curitiba/PR, foi recebido para tratamento médico veterinário especializado.

Foi realizado, então, um exame físico completo, estando o animal sob anestesia geral por meio do protocolo de administração de zoletil 50 na dose de $5 \mathrm{mg} / \mathrm{kg} / \mathrm{IM}$, associado ao butorfanol no volume de $0,12 \mathrm{~mL} / \mathrm{SC}$, quando foi constatado que o animal se apresentava com prostração, anorexia, secreção nasal bilateral, paresia de membros pélvicos e lesões em pele severamente contaminadas. Havia uma lesão de cinco centímetros de diâmetro em dorso, localizada em região pélvica, bastante profunda e com um odor fétido, além também de algumas lesões perfurantes com secreção purulenta na região da cauda, sugestivo de ataque por carnívoros. A auscultação cardiorrespiratória mostrou-se dentro dos parâmetros normais para a espécie. Não foi detectada crepitação em nenhum segmento vertebral durante o exame ortopédico realizado. $\mathrm{O}$ paciente demonstrou resposta à dor profunda em membros pélvicos.

Devido à inexistência de diagnóstico preciso até o momento do exame clínico, o animal foi encaminhado para exames radiográficos.

Inicialmente foram feitas radiografias simples, de dupla exposição - ventrodorsal e laterolateral -, de todos os segmentos vertebrais, não sendo observadas quaisquer alterações. Dessa maneira, optou-se por realizar um exame contrastado, a mielografia. Para a realização do exame, o paciente estava sob anestesia geral (Zoletil 50$5 \mathrm{mg} / \mathrm{kg} / \mathrm{IM})$, e a pele da região da articulação atlanto-occipital foi preparada com tricotomia e assepsia cirúrgica correta. Não foi possível efetuar a entubação do paciente, porém ele se encontrava sob monitoramento cardiorrespiratório. Como não foram encontradas doses sugestivas para o uso de contraste em ouriços, utilizou-se, então, experimentalmente, a mesma dose indicada para cães de $0,3 \mathrm{~mL} / \mathrm{kg}$ de peso corporal (Tudury et al., 1997; Slatter, 1998; Kealy e McAllister, 2005), totalizando, assim, $0,4 \mathrm{~mL}$ de ioversol $320 \mathrm{mg} \mathrm{I} / \mathrm{mL}$ (Tudury et al., 1997).

Uma agulha $25 \mathrm{mmx} 0,7 \mathrm{~mm}(22 \mathrm{G} 1)$ foi inserida na cisterna magna, com o animal posicionado em decúbito lateral e a saída de líquido cefalorraquidiano (LCR) constatou o correto 
posicionamento dela. Após a saída de quatro gotas de LCR, procedeu-se à administração de $0,4 \mathrm{~mL}$ do meio de contraste à base de iodo não iônico, ioversol, lentamente, por um período de dois minutos. Após tal procedimento, o animal teve sua cabeça e pescoço elevados para que o contraste fluísse caudalmente no interior do canal medular. Ao término da injeção do meio de contraste, iniciaram-se, então, exames radiográficos em intervalos de cinco minutos cada. Foram obtidas duas incidências radiográficas, decúbito lateral e dorsal, dos segmentos vertebrais cervical, torácico e toracolombar. Foi evidenciado desvio dorsal da coluna de contraste na altura da sétima vértebra torácica $\left(\mathrm{T}_{7}\right)$, causado por compressão em região ventral desta vértebra. A coluna de contraste não alcançou a região da cauda equina (Fig. 1).

O animal, após o procedimento radiográfico, foi monitorado até o seu completo retorno da anestesia efetuada e não apresentou qualquer indício de alteração de comportamento.

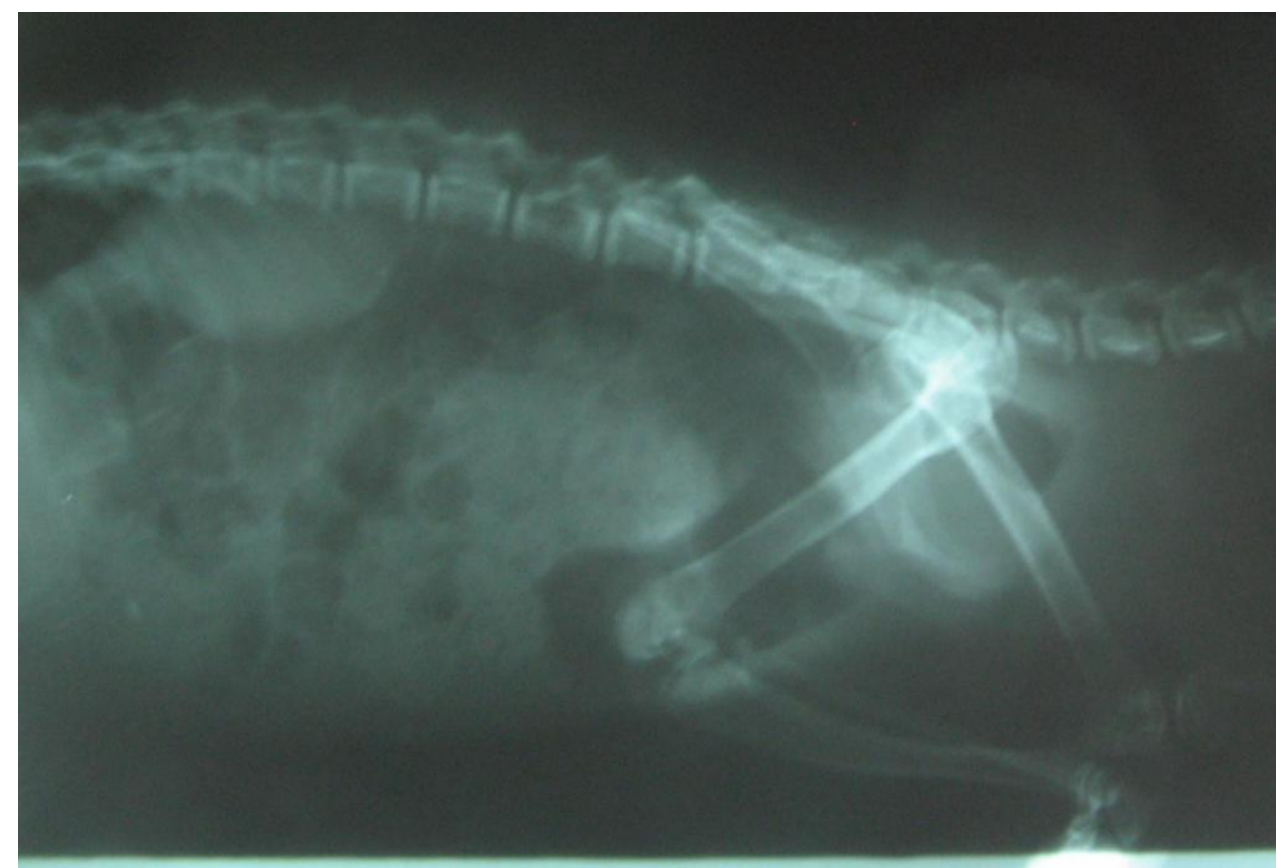

Figura 1. Mielografia mostrando que a coluna de contraste não alcançou região da cauda equina.

\section{DISCUSSÃO}

Segundo Cooper (1996), as interações intraespecíficas e interespecíficas podem resultar em lesões traumáticas em animais de vida livre, como em casos de predação, defesa de território e atividades relacionadas com o acasalamento, fato esse observado no presente trabalho, em que o animal relatado apresentava lesões sugestivas de interação interespecífica, provavelmente advindas de alguma espécie de carnívoro. Esse autor ainda citou que os animais afetados por injúrias traumáticas geralmente exibem sinais de dor com feridas externas visíveis ou palpáveis, podendo também ocorrer paresia e paralisia no animal. E como forma de diagnóstico, além de uma completa avaliação à procura de escoriações, hematomas ou outros indícios de trauma, exames radiográficos ou mesmo outras técnicas imaginológicas podem ajudar no fechamento de um correto diagnóstico para certos tipos de trauma, o que corrobora com os achados no presente relato.

Os resultados radiográficos do exame contrastado evidenciaram a existência de lesão intramedular, tendo como possível causa o edema ocasionado pela hemorragia intramedular secundária ao trauma, conforme descreve Knipe (2007). Entretanto atualmente a mielografia está sendo substituída pela ressonância magnética devido a sua maior precisão e à ausência de eventuais efeitos colaterais associados com a administração do meio de contraste iodado (De 
Lahunta e Glass, 2007), bem como pela tomografia computadorizada, a qual é uma ferramenta muito útil na avaliação de estruturas como a cabeça e a coluna vertebral, pois minimiza os efeitos de sobreposição de tecido ósseo e de tecidos moles, os quais ocorrem nas radiografias (Evans e Souza, 2010).

O tratamento paliativo para a lesão descrita consiste em uma combinação de restrição de espaço, reabilitação física e a administração de analgésicos e anti-inflamatórios. $\mathrm{O}$ uso deste último ainda é um assunto de considerável debate, existindo casos de melhora e de piora quando tais drogas são prescritas (Srugo et al., 2010).

Como alguns casos de fatalidades traumáticas envolvendo animais selvagens não podem ser tratados de maneira satisfatória, a eutanásia é uma opção de escolha. Os fatores que devem ser levados em consideração incluem a severidade das lesões, a espécie animal, as exigências e a perspectiva a longo prazo (Cooper, 1996). No presente caso, a eutanásia foi realizada devido às condições desfavoráveis de tratamento, recuperação e sobrevivência do paciente em seu hábitat.

\section{CONCLUSÕES}

A utilização da mielografia em Sphiggurus villosus (ouriço-cacheiro) mostrou ser bastante válida e eficiente, apresentando como vantagens a maior precisão para delimitar e localizar a lesão presente na coluna vertebral.

\section{REFERÊNCIAS}

BARONE, G.; LISA, S.Z.; FRANCES, S.S.; SHELDON, A.S. Risk factors associated with development of seizures after use of iohexol for myelography in dogs: 182 cases. J. Am. Vet. Med. Assoc., v.220, p.1499-1502, 2002.

COOPER, J. E. Physical Injury. In: FAIRBROTHER, A.; LOCKE, L.N.; HOFF, G.L. (Eds). Noninfectious Diseases of Wildlife. 2.ed. London: Manson Publishing, 1996, p.157172.

DE LAHUNTA, A.; GLASS, E. Small animal spinal cord disease. In: __. (Eds). Veterinary Neuroanatomy and Clinical Neurology. 3.ed., St. Louis: W.B. Saunders, 2007. p.243-285.
EVANS, E.E.; SOUZA, M.J. Advanced Diagnostic Approaches and Current Management of Internal Disorders of Select Species (Rodents, Sugar Gliders, Hedgehogs). Vet. Clin. Exot. Anim., v.13, p.453-469, 2010.

KEALY, J.K.; McALLISTER H. Radiologia $e$ Ultrassonografia do Cão e do Gato. 3.ed. Barueri: Manole, 2005. 436p.

KNIPE, M.F. Principles of Neurological Imaging of Exotic Animal Species. Vet. Clin. Exot. Anim., v.10, p.893-907, 2007.

LANGE, R.R; SCHMIDT, E.M.S. Rodentia Roedores Silvestres (Capivara, Cutia, Paca, Ouriço). In: CUBAS, Z.S.; SILVA, J.C.R.; CATÃO-DIAS, J.L. (Eds.). Tratado de Animais Selvagens - Medicina Veterinária. São Paulo: Roca, 2007. p.475-491.

OLIVEIRA, J.A.; BONVICINO, C.R. Ordem Rodentia. In: REIS, N.R.; PERACCHI, A.L.; PEDRO, W.A.; LIMA, I.P. (Eds). Mamíferos do Brasil. Londrina: Nélio R. Dos Reis, 2006. p.347-406.

PINTO, A.C.B.C. F. Radiologia. In: CUBAS, Z.S.; SILVA, J.C.R.; CATÃO-DIAS, J.L. (Eds.). Tratado de Animais Selvagens - Medicina Veterinária. São Paulo: Roca, 2007, p.896-919.

SLATTER, D. Manual de Cirurgia de Pequenos Animais. SP: Manole, v.1, p.582-590, 1998.

SRUGO, I.; CHAI, O.; YAAKOV, C. et al. Successful medical management of lumbar intervertebral disc prolapse in a ferret. J. Small Anim. Pract., v.51, p.447-450, 2010.

THRALL, D.E. Textbook of Veterinary Diagnostic Radiology. 4.ed. Philadelphia: Saunders, 2002. 758p.

TUDURY, E.A.; ARIAS, M.V.B.; CAMARGO, P.L. et al. Meio de contraste ioversol em neuroradiologia canina. Cienc. Rural, v.27, 1997.

WILSON, D.E.; REEDER, D.M. Mammals Species of the World - A Taxonomic and Geografhic Reference. 2.ed. Smithsonian Institution, 1993. 1206p. 